\title{
EXTENSIONS GENERATED BY CLOSED SETS
}

\author{
M. E ABD EL-MONSEF, A. M. KOZAE AND A. A. ABO-KHADRA
}

\begin{abstract}
From the nonempty collection of all closed sets $(Y)$ of any topological space $(X, \tau)$, Schmidt generates a topological space $(Y, \mathcal{U})$. In this paper, we give some properties of this topological space. We determined when $(f,(Y, \mathcal{U}))$ is an extension of $(X, \tau)$. Also we give some separation properties. This paper leads us to unsolved problem mentioned at the end of it.
\end{abstract}

\section{Introduction}

Throughout the present paper $X$ means a topological space on which no separation axioms are assumed unless explicitly stated. Let $A$ be a subset of $X$, the complement of $A$ will be denoted by $\sim A$. Let $(X, \tau)$ and $(Y, \mathcal{U})$ be topological spaces, and $S$ be a dense subset of $Y$, if there exists a homeomorphism $f:(X, \tau) \rightarrow\left(S, \mathcal{U}_{S}\right)$, then the pair $(f,(Y, \mathcal{U}))$ is called an extension of $(X, \tau)$. If $(Y, \mathcal{U})$ is compact, then the extension $(f,(Y, \mathcal{U}))$ is called a compactification of $(X, \tau)$. Aspace $X$ is called cid [3] if every countable infinite subspace of $X$ is discrete. Aspace $(X, \tau)$ is $R_{1}[1]$ iff for $x, y \in X$ such that $\overline{\{x\}} \neq \overline{\{y\}}$ there exist disjoint open sets $U$ and $V$ such that $\overline{\{x\}} \subset U$ and $\overline{\{y\}} \subset V$.

Theorem 1.1. [3] (a) A space $X$ is cid iff every countable infinite subset is closed.

Received April 8, 1992.

1990 Mathematics Subject Classification: 54A10, 54D10, 54D15, 54025.

Keywords and Phrases: extension of spaces, compactification, cid spaces, normal space. 
(b) Any infinite cid space is $T_{1}$.

\section{General Properties}

Definition 2.1. [4] Let $(X, \tau)$ be a topological space, and $Y=\tau^{c}-\{\phi\}$. For each open subset $U$ of $X$, let $U^{*}=\{F \in Y: F \subset U\}$. $Y$ with the topology generated by the collection $\beta=\left\{U^{*}: U \in \tau\right\}$ is denoted by $\mathcal{U}$. It is easy to prove that the collection $\beta$ form a base for a topology $\mathcal{U}$ on $Y$. In [2] This new topology were studied especially subspaces.

Example 2.1. Let $X=\{a, b, c\}$, and $\tau=\{X, \phi,\{c\},\{a, b\}\}$. Then $Y=$ $\{X,\{a, b\},\{c\}\}$ and $\mathcal{U}=\{Y, \phi,\{\{c\}\},\{\{a, b\}\},\{\{c\}\},\{\{a, b\}\}\}$.

Remark 2.1. If in $(X, \tau)$ each proper open set contain no closed set then $(Y, \mathcal{U})$ is indiscrete, for example the excluded point topology [6]. The following theorem determines some properties of the elements of the collection $\beta$ in any space.

Theorem 2.1. Let $(X, \tau)$ be a space, then the following statments are hold for all $U$ and $V \in \tau$

(i) $(U \cap V)^{*}=U^{*} \cap V^{*}$,

(ii) $U^{*} \subseteq V^{*}$ iff $U \subseteq V$,

(iii) $U^{*} \neq V^{*}$ iff $U \neq V$,

(iv) $(U \cup V)^{*}=U^{*} \cup V^{*}$, if $X$ is cid and $T_{1}$-space.

Proof. (i), (ii), and (iii) are obvious.

(iv) Let $F_{1} \in(U \cap V)^{*}=\{F \in Y: F \subset U \cup V\}$. Then there are two cases:

(a) $F_{1} \subset U$, or $F_{1} \subset V$, or $F_{1} \subset U \cap V$, in each case, we have $F_{1} \in U^{*} \cup V^{*}$, and hence $(U \cup V)^{*} \subset U^{*} \cup V^{*}$. Thus $(U \cup V)^{*}=U^{*} \cup V^{*}$.

(b) $F_{1}=F_{U} \cup F_{V}$, where $F_{U} \subset U$, and $F_{V} \subset V$. Since $X$ is cid, we have that $F_{1}$ is countable, and $F_{U}, F_{V}$ are countable and closed sets. Thus $F_{1} \in U^{*} \cup V^{*}$, and $(U \cup V)^{*}=U^{*} \cup V^{*}$. 
Remark 2.2. In general $(U \cup U)^{*} \neq U^{*} \cup V^{*}$ and we cannot drop the hypothesis " $T_{1}$ " in Theorem 2.1 (iv) as the following example shows.

Example 2.2. Let $X=\{a, b, c, d, e\}$, and $\tau=\{X, \phi,\{a, b, c\},\{b, c, d\},\{b$, $c, e\},\{b, c\},\{a, b, c, d\},\{a, b, c, e\},\{b, c, d, e\}\}$. Let $U=\{b, c, d\}$ and $V=\{a, b, c\}$. Then $U^{*} \cup V^{*}=\{\{a\},\{d\}\}$, and $(U \cup V)^{*}=\{\{a\},\{d\},\{a, d\}\}$.

\section{Extension}

In the following we shall answer on the question. When $(f,(Y, \mathcal{U}))$ is an extension of $(X, \tau)$.

Theorem 3.1. Let $(X, \tau)$ be a $T_{1}$-space, then $(Y, \mathcal{U})$ is also $T_{1}$.

Proof. Let $F_{1}, F_{2} \in Y$, and $F_{1} \neq F_{2}$, then there are two cases:

(a) There exists $x \in F_{1}, x \in F_{1}, x \notin F_{2}$, which implies that for each $y \in F_{2}$, $x \neq y$.

(b) There exists $y \in F_{2}, y \notin F_{1}$, which implies that for each $x \in F_{1}, x \neq y$. Since $(X, \tau)$ is $T_{1}$, we have for all $y \in F_{2}$, there exists $U_{y} \in \tau$ and $x \notin U_{y}$. This is implies that $\{x\} \not \subset U_{y}^{*}$ and $\{x\} \not \subset \bigcup_{y \in F_{2}} U_{y}^{*}=W$, where $W$ is open in $(Y, \mathcal{U})$.

Hence, $F_{2} \in W$ and $F_{1} \notin W$. Similarly $F_{1} \in H$, where $H$ is open in $(Y, \mathcal{U})$ and $F_{2} \notin H$. Hence $(Y, \mathcal{U})$ is a $T_{1}$-space.

Theorem 3.2. Let $(X, \tau)$ be a $T_{1}$-space, and we take $f:(X, \tau) \rightarrow(Y, \mathcal{U})$ such as $f(x)=\{x\}$, for each $x \in X$, then: $(f,(Y, \mathcal{U}))$ is an extension of $(X, \tau)$.

Proof. Since $X$ is $T_{1}$, and $Y=\tau^{c}-\{\phi\}$. We have that $Y=X \cup\{F \in Y: F$ is not single subset\}. We have the following:

(i) $X \subset Y$.

(ii) $f(X)$ is dense in $(Y, \mathcal{U})$. To prove this, Let $W$ be any open set of $\mathcal{U}$, then $W=\cup\left\{U_{i}^{*}: i \in I\right\}$, where $U_{i}^{*} \in \beta$, and $I$ is an arbitrary index set. Then there exists at least $U_{i_{0}}^{*} \in W$ such that $\{x\} \in U_{i_{0}}^{*}$. Thus $f(X) \cap W \neq \phi$.

(iii) The function $f:(X, \tau) \rightarrow\left(f(X), \mathcal{U}_{f(X)}\right)$ is $1-1$ and onto. 
(iv) It remains to prove that $f$ is a homeomorphism. Let $G \in \tau$, and $\{x\} \in$ $f(G)$, then $x \in G$, and $\{x\} \in G^{*} \in W$, where $W \in \mathcal{U}$. Thus $\{x\} \in U \cap f(x)$, and $f(G) \subset U \cap f(X)$. Conversely, if $\{x\} \in U \cap f(x)$, there exists $G^{*} \in U \cap f(X)$ such that $x \in G \in \tau$, which implies that $\{x\} \in f(G)$. Hence $U \cap f(X) \subset f(G)$. Thus $f(G)=U \cap f(x)$. Also $f^{-1} f(G)=f^{-1}(U \cap f(x))=G$.

\section{Separation $\mathbb{P r o p e r t i e s}$}

Theorem 4.1. $(X, \tau)$ is regular iff $(Y, \mathcal{U})$ is $T_{2}$.

Proof. First, if $(X, \tau)$ is regular, then the proof that $(Y, \mathcal{U})$ is a $T_{2}$-space is similar to the proof in Theorem 3.1. Second, let $(Y, \mathcal{U})$ is $T_{2}, F$ is closed set, and $x \notin F$. We assume that $x \in F^{\prime}$, where $F \neq F^{\prime}$, then there exist $U^{*}$ and $V^{*} \in \mathcal{U}$ such that $F \in U^{*}$ and $F^{\prime} \in V^{*}$ such that $U^{*} \cap V^{*}=\phi$. Thus $F \subset U$ and $F^{\prime} \subset V$ and $U \cap V=\phi, x \in V$. Hence $(X, \tau)$ is regular.

Theorem 4.2. (a) If $(X, \tau)$ is $T_{4}$, then $(Y, \mathcal{U})$ is $T_{2}$. (b) If $(Y, \mathcal{U})$ is $T_{2}$, and, $(X, \tau)$ compact space, then $(X, \tau)$ is $T_{4}$.

Proof. (a) Let $F_{1}, F_{2} \in Y$ such that $F_{1} \neq F_{2}$, then there exist $U, V \in \tau$ such that $F_{1} \subset U, F_{2} \subset V$, and $U \cap V=\phi$. Hence, $F_{1} \in U^{*}$ and $F_{2} \in V^{*}$ such that $U^{*} \cap V^{*}=(U \cap V)^{*}=\phi$.

(b) By using Theorem (4.1), the prove is obvious.

Theorem 4.3. If $(X, \tau)$ is $R_{1}$, then $(Y, \mathcal{U})$ is $T_{4}$.

Proof. It is simillar to the prove of Theorem 4.2 (a)

Unsolved Problem. Under what conditions is the last extension $(f,(Y, \mathcal{U}))$ a compactification of $(X, \tau)$ ?

\section{Acknowledgement}

We would like to thanks the referee for valuable comments and suggestions. 


\section{References}

[1] A. D. Davis, "Indexed systems of neighborhoods for general topological spaces," The Amer. Math. Monthly, 68 (1961), 886-893.

[2] P. Marcello and R. Emanuele, "Further properties of a certain immersion into spaces of subsets," Boll. Un. Mat. Ital. A (6) 5 (1986), no. 3, 345-347.

[3] I. L. Reilly, M. K. Vamanamurthy, "On spaces in which every denumerable subspace is discrete," Math. Vesnik 38 (1986), 97-102.

[4] H. J. Schmidt, "Hyperspaces of quotient and subspaces," I. Hausdorff topological spaces. Math. Nacher 104 (1981), 271-280.

[5] H. J. Schmidt, "Hyperspaces of quotient and subspaces," II. Metrizable spaces. Math. Nachr. 104 (1981), 281-288.

[6] L. A. Steen and J. A. Seebach, "Counterexamples in topology," Springer-Verlag, New York 1978.

[7] W. J. Thron, "Topological structrs," Holt, Rinehart and Winston, Inc, New York (1966).

Department of Mathematics, Faculty of Science, Tanta University, Tanta, Egypt. 